\section{The effects of NICE guidelines on the management of third molar teeth}

\author{
L. W. McArdle ${ }^{1}$ and T. Renton ${ }^{2}$
}

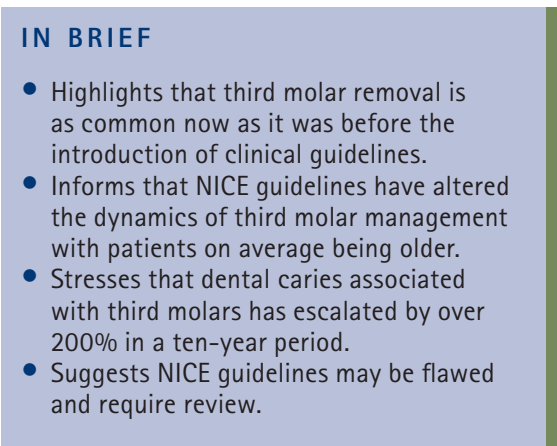

\begin{abstract}
Background Third molar surgery (TMS) is probably one of the most commonly performed surgical procedures undertaken in the NHS. In 2000, the National Institute of Clinical Excellence (NICE) introduced guidelines relating to TMS. These recommended against the prophylactic removal of third molars and listed specific clinical indications for surgery. The impact of these guidelines has not been fully evaluated and this research hopes to focus the effect of these guidelines over the last ten years. Methods Using data obtained from a variety of NHS databases such as HES (Eng \&t Wales), the NHSBSA and data from NHS Scotland, we looked at the age range of patients requiring third molar removal and the number of patients having third molars removed in both primary and secondary care environments from 1989 to 2009. In addition we looked at the clinical indications for TMS activity in secondary care. Findings The mean age of patients increased from 25 years in 2000 to 32 years in 2010, with the modal (most common) age increasing from 26 to 29 years. After the introduction of clinical guidelines the number of patients requiring third molar removal in secondary care dropped by over 30\%, however, since 2003 the number of patients has risen by 97\%. There is also a significant increase in caries as an indication for third molar removal. Conclusions More patients are requiring third molar removal with an increasing number of patients having caries related to their third molars. Patients are, on average, older confirming that the removal of third molars is shifting from a young adult population group to an older adult population group. NICE guidelines did appear to have contributed to a fall in the volume of third molars removed within the NHS post 2000. However, concluding that this reduction demonstrates the success of NICE's guidance would be a premature assumption. The number of patients now requiring third molar removal is comparable to that of the mid 1990s. NICE has influenced the management of patients with third molars but this has not resulted in any reduction in the number of patients requiring third molar removal. Coding and data collection for third molars is not uniform, leading to potential misrepresentation of data. This perhaps raises the issue that an improved universal coding system is required for the NHS and that the NICE guidelines need review.
\end{abstract}

\section{INTRODUCTION}

Impacted third molars (wisdom teeth) are one of the most common developmental conditions that affect humans. It occurs due to a failure of proper eruption of the third molar tooth resulting in impaction of the tooth against adjacent teeth, alveolar bone, the surrounding mucosal soft tissue or combination thereof (Fig. 1). The impaction is defined in relation to the geometric angle of impaction such as mesio-angular, disto-angular, vertical and horizontal.

${ }^{1 *-2}$ Consultant Oral Surgeon, King's College London Dental Institute

${ }^{*}$ Correspondence to: Dr Louis W. McArdle Email: Iwmcardle@msn.com

\section{Refereed Paper}

Accepted 14 May 2012

DOI: 10.1038/sj.bdj.2012.780

${ }^{\circ}$ British Dental Journal 2012; 213: E8
If the tooth cannot fully erupt then its impaction will also be defined as partial, where some of the tooth has erupted into the oral cavity; or complete, where the tooth is buried and completely unerupted. The most common third molar tooth to be impacted is the mandibular third molar followed by the maxillary third molar. Third molar development tends to be bilateral although failure of the third molar to develop, either unilaterally or bilaterally, is not uncommon. Impacted third molars can cause a host of clinical problems that may necessitate the removal of the tooth to facilitate dental health.

Third molar surgery (TMS) is one of the most commonly performed surgical procedures undertaken in secondary care within the NHS. When combined with out-patient procedures undertaken in both secondary

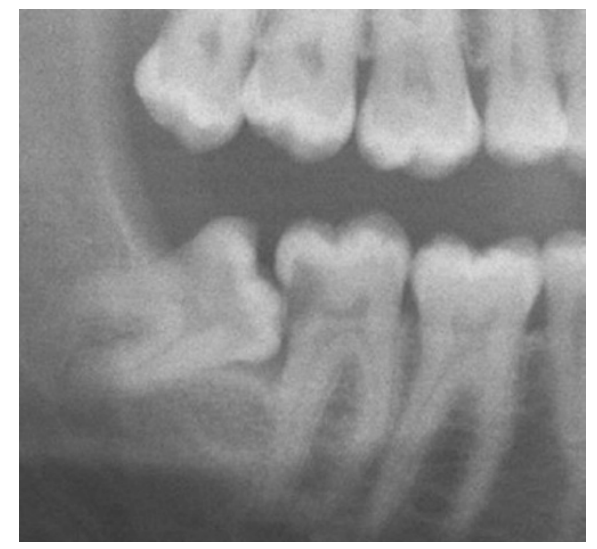

Fig. 1 Mesio-angular impacted third molar with caries in-situ and causing caries to the second molar. Lower right side

care and primary dental care it probably rates as the most common surgical procedure undertaken in the whole of the NHS. The presence of an impacted third molar is a developmental condition and is 
recognised as such by the World Health Organisation within the definitions of the International Coding for Diseases (ICD-10). ${ }^{1}$ It is accepted that the removal of a diseased or symptomatic third molar tooth will alleviate pain and symptoms and improve the oral health and function for patients. ${ }^{2}$

The National Institute for Health and Clinical Excellence (NICE) issued guidance on the management of third molars in $2000 .^{3}$ They surmised that up to $40 \%$ of third molars removed had no clinical indication for removal and that the practice of prophylactic removal should be discontinued within the NHS which, in turn, could generate an annual saving of $£ 5$ million for the NHS.

The impact of NICE's guidelines has not been properly evaluated and NICE's guidance remains the same. This paper aims to evaluate the impact of NICE guidance on the change in clinical practice based on the numbers of patients undergoing third molar surgery, the indications for surgery and the changes in patient demographics over the last 20 years, prior to and after the introduction of NICE guidelines.

NICE guidelines on the management of third molars followed the introduction of other notable clinical guidelines for third molar management. In 1979, the National Institute of Health in the USA issued their guidelines on the management of third molars partly as a result of comment by medical insurance companies that third molars where being removed unnecessarily without any evidence-based clinical indication. ${ }^{4}$ In 1991, the American Association of Oral and Maxillofacial Surgeons introduced their guide and parameters of care document. ${ }^{5}$ The first UK evidence-based guide to third molar management was issued by the Faculty of Dental Surgery of the Royal College of Surgeons of England in 1997 and most recently the guidance issued by NICE in 2000, complemented by the Scottish Intercollegiate Guidelines Network (SIGN) guidance also in 2000.3,6,7

NICE's main advice relates to the indications for removal of third molars and that the routine practice of prophylactic removal of pathology-free impacted third molars should be discontinued. Specifically, the clinical indications for the removal of impacted third molars should be limited to patients with evidence of disease (Fig. 2).

\section{METHODS}

To determine the trend in the number of patients in the UK having third molars removed over the last 20 years. Data was collated from the NHS Hospital Episodes Statistics (HES) database and the NHS Business Support Agency (NHSBSA). ${ }^{8,9}$ In addition data was also collated from the Information Services Division (ISD) of the NHS in Scotland.

HES is an NHS database that records secondary care hospital-based activity in England and Wales. Included in the database are all patient diagnostic codes based on the World Health Organisation, WHO ICDN-10 codes and surgical activity codes based on the OPCS coding system. For England and Wales, primary dental care is evaluated by the NHS BSA who, in 2005, took over the role that the Dental Practice Board (DPB) had previously. The DPB recorded all dental procedure codes for each individual dentist with a General Dental Services (GDS) contract in the UK. This allowed treatment profiles of dentists to be collated and analysed. Within both of these databases, records exist for patients who have had third molars removed. Unfortunately, the NHSBSA stopped recording this data in 2006, as it was not part of their remit. From this data we can determine the relative level of third molar removal activity in the England and Wales. For Scotland the same primary care GDS and equivalent HES secondary care data is collated by the ISD of NHS Scotland. Figures for Scotland are reviewed separately to allow comparison post 2005 and the introduction of the new dental contract in England and Wales. In addition to these data, we have assessed HES data for the change of demographic profiles of patients and reviewed the clinical indication for the removal of third molars.

It is important to appreciate that the data recording by HES and the DPB/NHSBSA is different. Patients may have more than one third molar removed during a course of treatment and if one does not appreciate the nuances of data recording by these systems then the interpretation could be confusing. HES records the number of patients that have had one or more third molars removed whereas the DPB records the actual number of third molars removed. Consequently from HES data we do not know how many third molars

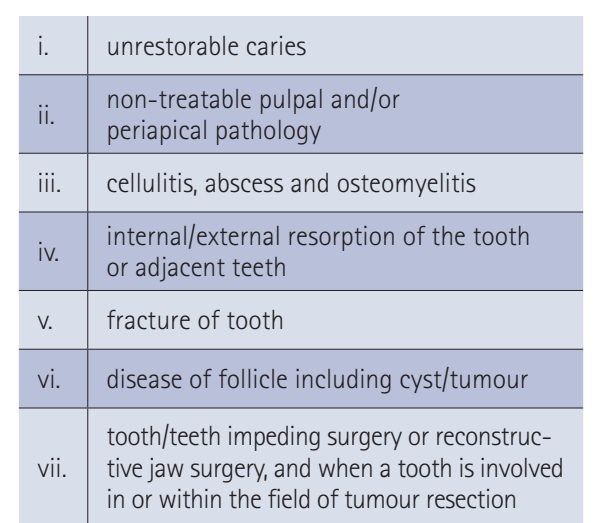

Fig. 2 NICE's clinical indications for the removal of third molar teeth

were actually removed and conversely from the DPB data we do not know the actual number of patients who had third molars removed. Because of this, more third molars could be being removed in secondary care, as the data would allude to, and conversely the numbers of third molars being removed in primary care does not reflect the total number of patients and this has to be taken into consideration in collating and analysing the data.

\section{RESULTS}

\section{HES finished consultant episodes}

Data recorded by HES relates to the number of patients who have been admitted to hospital for either a day-case or in-patient procedures under either GA or IV sedation. In general, patients who have had third molars removed under local anaesthesia on an out-patient basis do not get included in HES data as this activity is recorded as an anonymous out-patient appointment and not as an out-patient surgical activity as with other surgical specialties. More recently, however, this type of activity has begun to be recorded but the true level of out-patient third molar removal is not known, and as such these out-patient figures may significantly underestimate the actual number of patients having third molars removed.

From the HES data, ${ }^{8}$ approximately 50,000 people per annum in England and Wales had third molar teeth removed in the early period of the 1990s (Fig. 3). This number rose to 70,000 by the mid 1990s and averaged approximately 60,000 patients per year for the whole of that decade. In the first half of the 2000s patient numbers started to decline significantly 


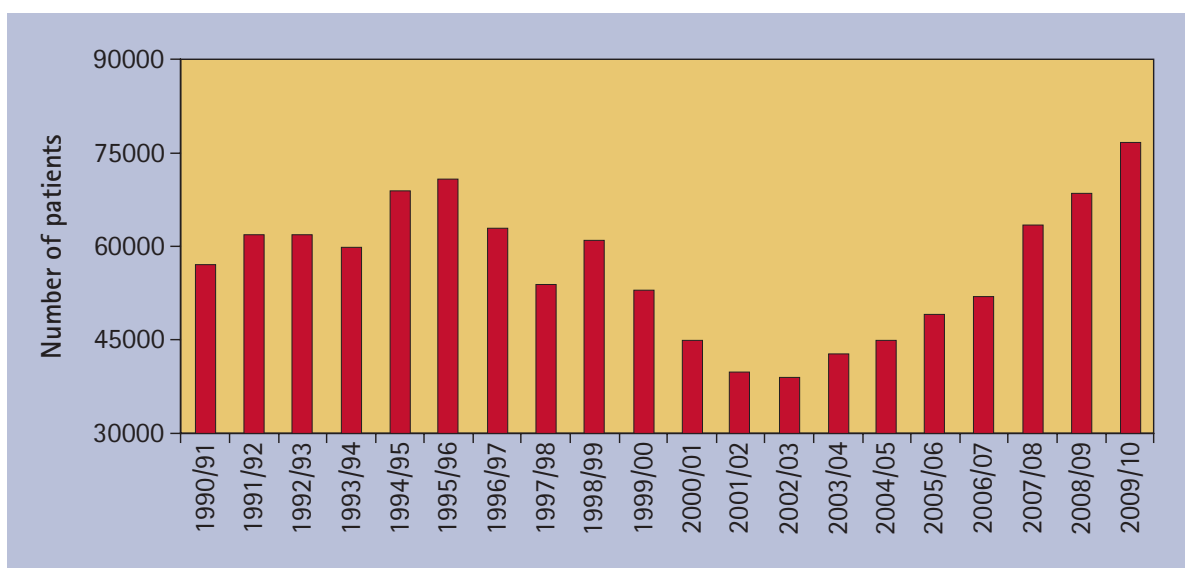

Fig. 3 Patients requiring third molar removal in secondary care: England and Wales

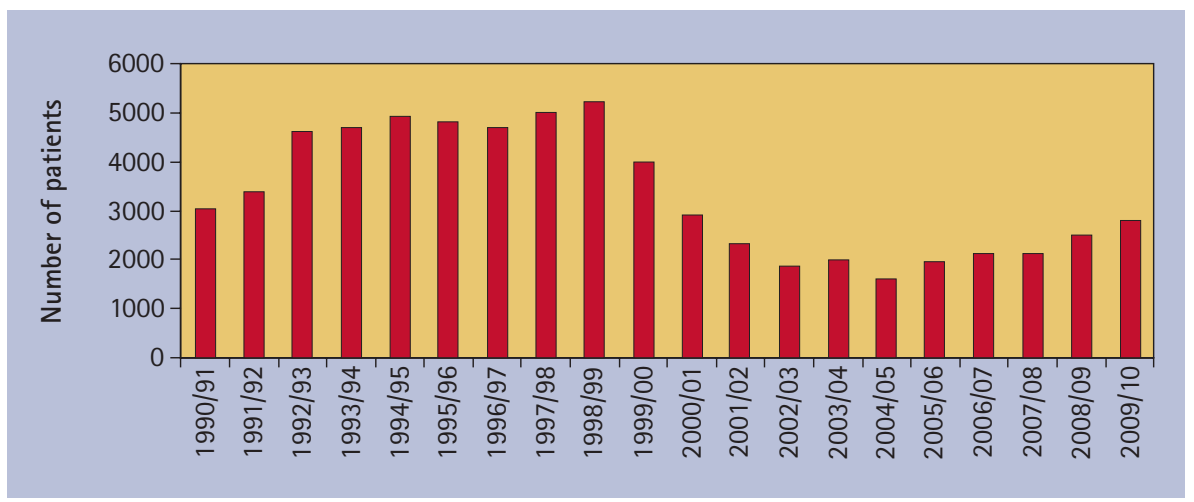

Fig. 4 Patients requiring third molar removal in secondary care: NHS Scotland

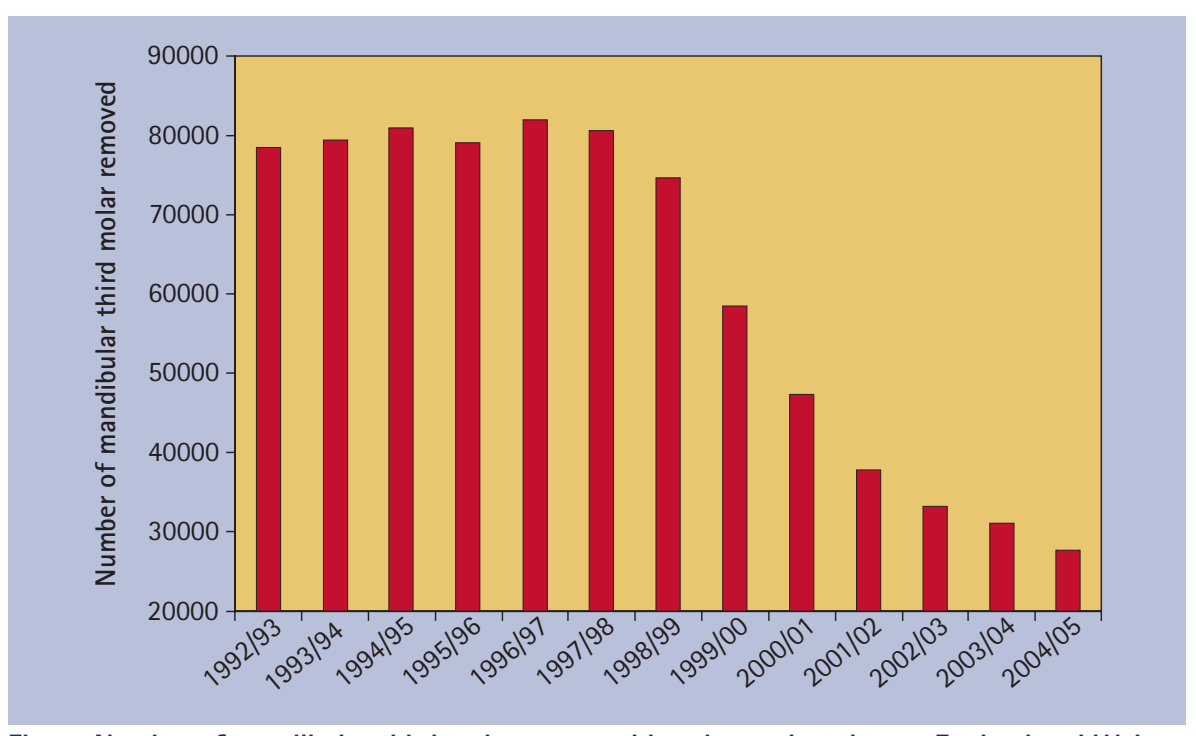

Fig. 5 Number of mandibular third molars removed in primary dental care: England and Wales

and by 2003 HES data suggests that less than 40,000 patients per annum were having third molar treatment undertaken in a hospital setting as either in-patient or day-case procedures: a reduction on the 1990s average of over 30\%. ${ }^{8}$

From the mid 2000s onwards, the recording of a small amount of out-patient activity has been included in HES data along with in-patient and day-case data. Over the latter 5 years of the 2000s the number of patients having third molars removed had increased to almost 77,000 patients per annum in 2009/10 (65,000 in-patient/ day-case; 12,000 out-patient). This equates to an approximate increase of $67 \%$ of inpatient and day-case activity recorded in the secondary care sector but a 97\% increase in all recorded patient activity. By 2009/10 patients having third molars removed in a hospital setting was most notably at its highest level for 20 years. ${ }^{8}$
In Scotland, figures for in-patient/ day-case activity follow a similar trend to England and Wales (Fig. 4). For most of the 1990s just under 5,000 cases of third molar removal were undertaken per annum. Subsequent to the introduction of the SIGN guidelines a similar trend in the number of cases per annum was noted, dropping to approximately 1,600 cases per annum by 2005: a reduction of approximately 70\%. Post 2005, however, a steady year-by-year increase was noted and by 2009/10, the numbers of patients had increased to approximately 2,800 cases: an increase of 67\% from the low of 2004/5.

\section{NHSBSA/DPB 2204/2205 codes}

The NHSBSA/DPB records items of treatment of each patient rather than treatment episodes for patients. ${ }^{9}$ The Statement of Dental Remuneration (SDR) document provides the item of service codes used previously under the old General Dental Services Contract. ${ }^{10}$ SDR Codes 2204 and 2205 are third molar specific and although third molars would have undoubtedly been removed under other codes such as 2101 and 2201, these codes are not tooth specific and cannot be used to identify third molar activity. In addition, SDR codes 2204/5 can identify both mandibular and maxillary teeth separately. This data set is limited to exclusively mandibular third molars, as the combined total of both maxillary and mandibular third molars removed may not accurately reflect the actual number of patients having third molar surgery. By restricting our data set to exclusively mandibular third molars we get a less distorted perception of the actual number of patients. In addition, mandibular third molars tend to cause greater clinical problems, are more surgically complex and have greater postoperative morbidity, making them the focus for most published research.

For the period of 1992-2005 in England and Wales GDS, the trend in third molar activity follows a comparatively similar pattern to secondary care (Fig. 5). For most of the 1990s approximately 80,000 mandibular third molars were being removed per annum. Subsequent to 2000, the numbers of mandibular third molars removed declined steadily by over 60\% reaching a level of 28,000 per annum for 2004/5. Data for after this period is not available, as the NHSBSA no longer records it. 
In Scotland, data for third molar activity continues to be collated for general dental practice (Fig. 6). In the first half of the first decade of the new millennium, there was a 36\% fall in mandibular third molars removed, mirroring the yearly trend of reduction in England and Wales. After 2004, however, mandibular third molar removal progressively increased and by the end of the decade was $130 \%$ greater than at its lowest level of 2004. This trend cannot be properly compared with the post-2005 new General Dental Services (nGDS) trend in England and Wales but it does complement the upward trend of secondary care third molar activity seen in both England and Wales, and in Scotland, which saw an increase of $67 \%$ over the same time period for day-case/ in-patient procedures.

\section{Age}

HES data reports that the average age of patients requiring third molar removal within the NHS has increased over the last 20 years. In 1990, the average age of a patient having third molars removed as a day-case/in-patient procedure was 25 years. This mean age has steadily risen and now the mean age for patients having third molars removed as a day-case/ in-patient is 32 (Fig. 7). ${ }^{8}$

\section{Clinical indications for third molar removal in secondary care}

HES data also records the main clinical diagnosis for third molar removal (Fig. 8). ${ }^{8}$ The most common recorded OPCS-10 coding and indication for third molar removal relates to embedded and impacted teeth (K01.0/K01.1), paradoxically these are not a NICE indication for third molar removal. In 1995 embedded/impaction is recorded as the main diagnosis for approximately $70 \%$ of all third molars removed (Fig. 4). Over the next 15 years there was an increase in the proportion of caries or related peri-apical abscess (K02.9/K04.7) being recorded as the main diagnosis from less than 10\% in 1995 , rising to almost $30 \%$ by 2009 . In the same period, periodontitis (K05.2/K05.3), as a recorded main diagnosis, stayed at a relatively constant level at approximately $15 \%$. One notable anomaly from the coding system is that pericoronitis is coded as periodontal disease and not as a separately defined condition.

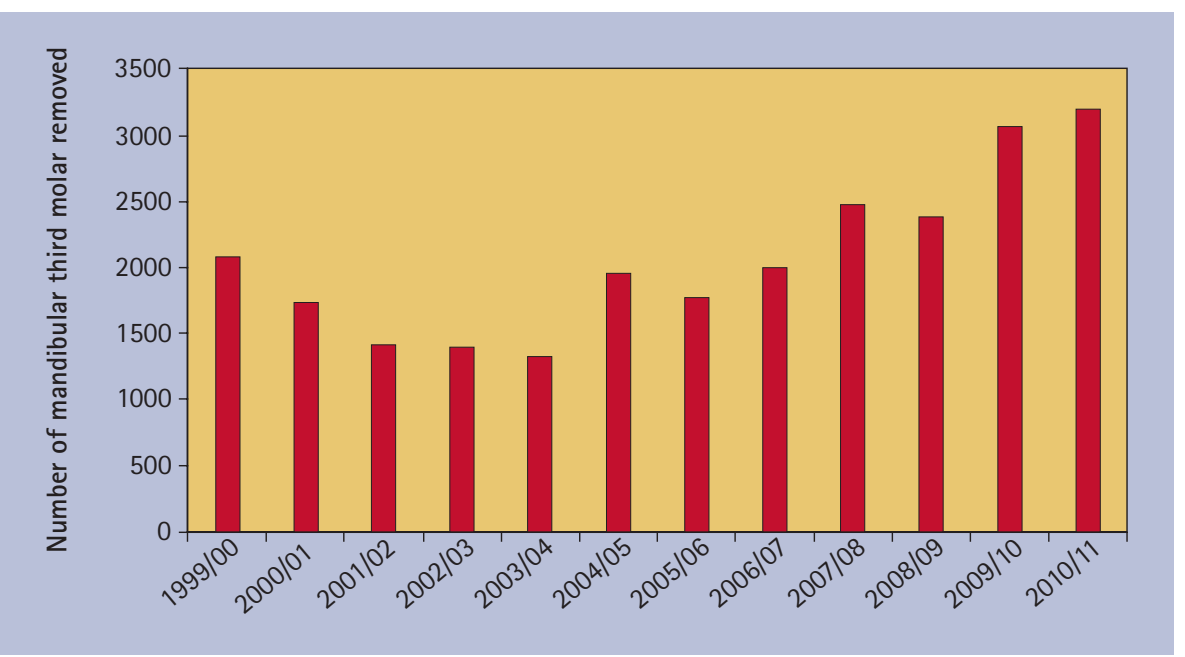

Fig. 6 Number of mandibular third molars removed in primary dental care: NHS Scotland

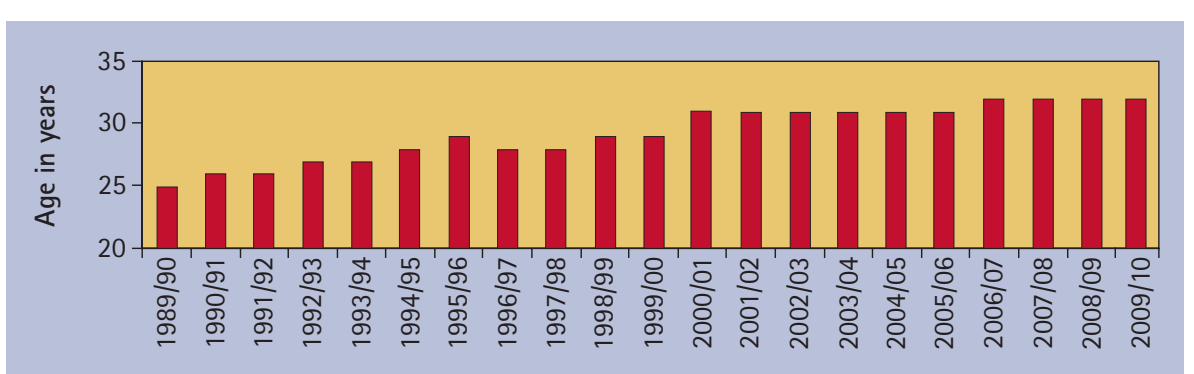

Fig. 7 Average age of patients requiring third molar removal in secondary care: England and Wales

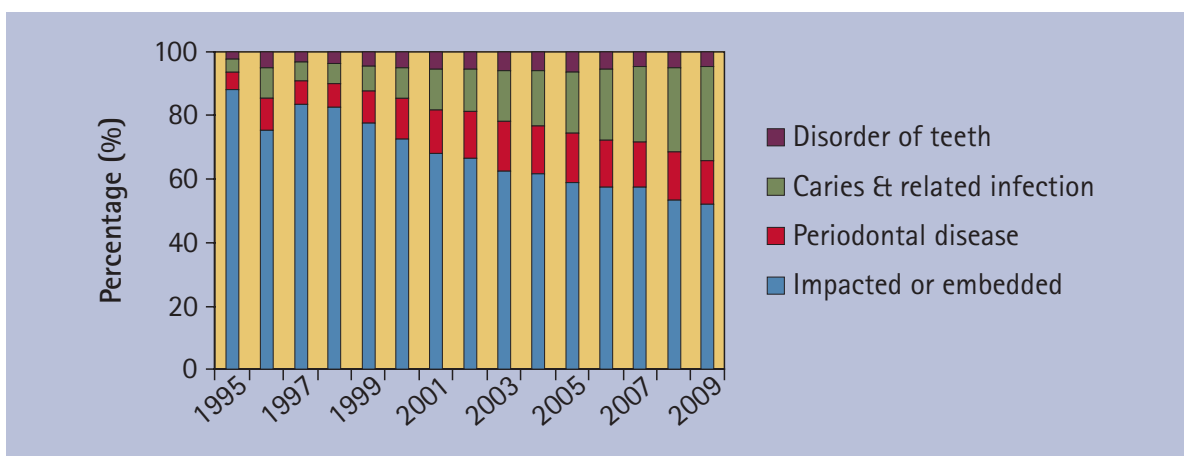

Fig. 8 Main clinical diagnosis for third molar removal in secondary care: NHS England and Wales

\section{DISCUSSION}

In 2004, a review of the impact that NICE guidance was having over a range of clinical interventions suggested that NICE had no discernable effect on the management of patients with third molar teeth. ${ }^{11}$ Data used by this study covered the period from 1995-2001, which observed a downward trend in third molar removal during this time. It was suggested that the downward trend in third molar removal had already begun as a consequence of guidance issued by the Royal College of Surgeons of England in 1997 and by the University of York in 1998. ${ }^{6,12}$ The sampling period of data was short and only included a single year after the introduction of NICE. Data prior to 1995, however, suggests that third molar removal was at a similar level for most of the 1990s with 1995 being the peak year within that decade. With the introduction of NICE's guidelines only occurring in 2000, it seems inappropriate to make this conclusion after only one year's worth of post-NICE data and a relatively short sample period.

It would appear that a decline in patients having third molars removed did start in the late 1990s with the introduction RCS(Eng) guidelines but our data suggests a significant reduction of third molar removal with the introduction of the NICE/ SIGN guidelines in 2000. ${ }^{11}$ It may be that 
the staggered introduction of the RCS(Eng) guidelines, the University of York's clinical effectiveness document and the NICE/SIGN guidance continued to reinforce the advice to the dental profession that third molars need not be removed. ${ }^{3,6,7,12}$

In general dental practice the numbers of third molars being removed reduced by over $50 \%$ by 2005 . For secondary care, data showed a $40 \%$ reduction in patients having third molars removed from a peak of 70,000 in the late 1990s down to 40,000 in the mid 2000s. On NICE's premise that $40 \%$ of all third molars being removed had no clinical indication for removal, then this data suggests that third molar guidelines were having the desired effect of reducing the number of third molars being removed and therefore reducing healthcare expenditure in this area.

Although data post-2005 is not available for the GDS in England and Wales, the data from Scotland and HES would suggest that this dip in primary care third molar activity in England and Wales might also be only temporary. Within secondary care in England and Wales, the fall in third molar activity mirrors primary care with numbers steadily falling from the late 1990s until 2002/3. Following this, however, the numbers for third molar removal in secondary care began to rise steadily with an increase from 39,000 patients per year in 2002/3 to almost 77,000 patients by $2009 / 10$ (65,000 in-patient/daycase; 12,000 out-patient): a total increase of $97 \%$ in 7 years. Furthermore, the data from NHS Scotland mirrors this increase in third molar activity by $67 \%$ in secondary care provision, and by 130\% for general dental services for the latter half of the 2000s.

The reasons for this increase in third molar activity in the latter half of the 2000s and the specific increase in secondary care activity may be explained by two possible hypotheses:

- The possible influence of the new General Dental Services (nGDS) contract in England and Wales in 2005

- A link between the increasing age of patients and the increasing incidence of caries related to third molars.

\section{The new General Dental Services}

The new General Dental Services (nGDS) contract was introduced in England and Wales in March 2006, whereas the GDS contract in Scotland has remained relatively unchanged. Dental treatment was previously provided at a cost per treatment item. The new GDS contract is based on the provision of treatment within treatment bands. At present, the treatment band generates the fee rather than the service item, or the quantity of different service items. Consequently, this results in NHS dentists receiving the same fee for a course of treatment that may include just a single restorative procedure and the exact same fee for a course of treatment for multiple restorative procedures, endodontic procedures and extractions. In this example the fee for a band 2 course of treatment may be in the region of £60-£70 and realistically this limited fee for multiple items of treatment does not necessarily cover the actual cost of providing the treatment.

As the contract does not offer remuneration based on the time the dentist spends with the patient, nor for the number nor complexity of treatment items, it has been suggested that dentists are unwilling to undertake some of the more complex treatment items on the NHS and are subsequently referring patients to other providers for treatment. ${ }^{13}$ The new GDS contract allows GDPs to refer patients for treatment that they themselves do not feel able to provide, whilst still claiming payment for the treatment. This may explain why referrals to hospital secondary care providers for oral surgery procedures, such as third molar extractions, has dramatically increased over the last five years. As such, oral surgery referrals to this teaching hospital in London have observed a 100\% increase since 2004/5. ${ }^{14}$ These observations may only be partly attributable to the increase in third molar removal in secondary care over the last five years, as the trend in secondary care third molar removal had already started to rise prior to the introduction of the new contract. From the trough of 2003 through to 2005 , the year before the new contract was introduced in England and Wales, third molar activity was already on the increase. In Scotland, this upward trend in third molar removal post-2005 is mirrored in both primary and secondary care settings, suggesting that the upward trend in England and Wales may be multi-factorial.

\section{Dental caries and age}

In relation to third molars, caries can affect the third molar itself or more significantly occur in the distal cervical area of the second molar tooth due to the mesio-angular impaction of the third molar against it. Caries is a disease that is relatively slow to develop compared with pericoronitis and as a consequence caries develops later in patients by comparison. ${ }^{15,16}$ From the HES data we have observed a significant increase in the number of patients requiring third molar removal. For the last ten years the recorded incidence of caries and its sequalae, as an indication for removal, has increased from less than $10 \%$ to almost $30 \%$ of all patients requiring third molar removal. ${ }^{8}$ Over the last ten years the recorded incidence of patients having third molars removed due to dental decay has increased by over $200 \%{ }^{8}$

It may be that the rapid dip in the number of third molar extractions in the early 2000s was due to a rigid interpretation and application of third molar guidelines and as such third molars were actively not removed. This may be true in cases of single or mild forms of pericoronitis or solely the presence of a partially erupted and impacted third molar that may have been used as the indication for removal pre-2000.

Third molars are not erupting later in life to account for the increase in mean age from 26 to 32 during the last 20 years. Third molars are being retained for longer, either as a result of lack of disease affecting younger patients, or a palliative approach to the management of third molar disease. Patients may be more inclined to be treated with antibiotics for recurring episodes of pericoronitis and thus avoid, or more likely, delay the removal of the third molar.

The fact that patients are retaining third molars later into life makes them more vulnerable to one of the problematic consequences of the oral environment: dental caries. The likelihood of this will be evident especially if the teeth are impacted, partially erupted and difficult to clean. Older patients with good dental health are more prone to having third molar teeth removed because of caries related indications such as DCC in the second molar. ${ }^{15-19}$ This data confirms that as patients have become older, dental caries has become an everincreasing problem related to third molars. This group of patients may be contributing to the rebound increase in the number of third molars being removed. 
With the mean age of patients increasing from 26 to 32 years of age, we see an increase in the number of patients requiring third molar removal due to caries. Over the age of 30, patients are more likely to have third molar teeth removed due to the effects of caries than those who are younger. ${ }^{15-18}$ Consequently, would it be reasonable to consider that any asymptomatic, partially erupted, impacted third molar, if retained, may ultimately cause patients clinical problems such as caries? If these problems are detrimental to the dental health of the patient then should we not consider defining the optimum time for removal - either at the time of disease presentation or even prior to the damage that the disease may cause - especially if the damage is related to the second molar in the form of distal cervical caries?

\section{ICD-10 coding}

There appears to be a lack of specificity in coding as it relates to studies such as this, which leads to problems in interpretation. Caries as a diagnosis is too non-specific for coding purposes. Caries associated with the third molar is an indication for third molar removal but distal cervical caries (DCC) on the second molar in the presence of a mesio-angular third molar is also an indication for third molar removal. Both of these clinical conditions appear to be on the rise in older age groups. ${ }^{15-18}$ The coding system does not allow us to isolate the nature of the decay and as a consequence limits our ability to interpret accordingly. Nonetheless, caries related to third molars is on the increase and its consequences have to be managed.

Pericoronitis is a definable clinical problem that affects partially erupted teeth and accounts for the removal of up to $60 \%$ of all mandibular third molar teeth. ${ }^{20-24}$ Pericoronitis is not, however, recognised by the WHO-ICDN coding system as a unique diagnosis and its classification as periodontal disease is erroneous. ${ }^{1}$ This flaw creates serious problems in accurate data interpretation. If databases are recording ICD-10 codes of K05.2 or K05.3 do they mean periodontal disease or pericoronitis? Local periodontal disease affecting the second molar tooth, in addition to periodontal disease of the third molar itself, are distinct indications for third molar removal, but to classify both pericoronitis and periodontal disease together is inappropriate and makes data interpretation difficult.

Impaction and embedded teeth are not in isolation an indication for third molar removal but merely an observation of the ectopic position that the tooth develops into. A tooth's abnormal position is a developmental anomaly and along with other developmental anomalies is defined within the ICD-10 coding system. ${ }^{1}$ This developmental anomaly ultimately accounts for the disease processes that affect impacted teeth, but recording the developmental anomaly rather than the disease that it predisposes, creates an imbalanced observation of the indications for third molar removal. In view of the actual HES incidence of impaction being comparable with the reported incidence of pericoronitis, it could be presumed that impaction is being recorded instead of pericoronitis. ${ }^{2,8,20-24}$

Accurate data collection in third molar studies and clinical coding systems is essential if data is to have any meaningful value. If the WHO ICD system is to be used for third molar data collection then it will require an overhaul to be fit for this purpose and to appropriately reflect the actual disease processes that afflict third molars.

\section{CONCLUSION}

With the introduction of clinical guidelines a decline in patients having third molars removed has occurred. This trend, however, has now been reversed and has steadily increased to pre-NICE levels. Any initial financial savings would have been short-term and with more patients attending secondary care for third molar procedures, costs are now greater than prior to the introduction of NICE. Patients are becoming older and more patients are experiencing caries as an indication for third molar removal even though the dental and oral health of the population continues to improve. ${ }^{25,26}$ Indeed patients with mandibular third molars who succumb to DCC on their second molar teeth have on average better dental health than their peers. ${ }^{16}$

It has been appreciated for some time that as the dental health of the population has improved, the early loss of first molar teeth in children and adolescents does not occur as frequently as before. ${ }^{27}$ Early loss of the first molar results in the forward drift and/or tipping of the second molar, creating space distally for the third molar to erupt unhindered and thus reduces the likelihood of impaction. Conversely, retention of the first molar restricts this space in the retro-molar area and no doubt contributes to the likelihood of impaction of the third molar tooth. ${ }^{27}$ The increase in third molar surgery seen over the last 30-40 years may not be due to inappropriate over-prescribing or prophylactic third molar removal but may, in fact, be due to the paradoxical consequence of improved dental health. It is likely that the number of patients requiring third molar removal will always be substantial.

1. World Health Organisation. International statistical classification of diseases and related health problems; 10th revision (ICD-10). Geneva: WHO, 2010.

2. Phillips $C$, Norman J, Jaskolka M et al. Changes over time in position and periodontal status of retained third molars. J Oral Maxillofac Surg 2007; 65: 2011-2017.

3. National Institute for Clinical Excellence. Guidance on the extraction of wisdom teeth. London: NICE, 2000. Online article available at http://egap.evidence.nhs.uk/guidance-on-the-extraction-of-wisdom-teeth-ta1 (accessed August 2012).

4. NIH consensus development conference for removal of third molars. J Oral Surg 1980; 38: 235-236.

5. American Association of Oral and Maxillofacial Surgeons (AAOMS). Report of a workshop on the management of patients with third molar teeth. J Oral Maxillofac Surg 1994; 52: 1102-1112.

6. Faculty of Dental Surgery of the Royal College of Surgeons of England. Current clinical practice and parameters of care: the management of patients with third molar teeth. London: Faculty of Dental Surgery RCS (Eng), 1997.

7. Scottish Intercollegiate Guidelines Network. Management of unerupted and impacted third molar teeth. SIGN, 1999. Online guideline available at http://www.sign.ac.uk/pdf/sign43.pdf (accessed August 2012)

8. Hospital Episode Statistics. HES online. Online information at www.hesonline.nhs.uk (accessed August 2012)

9. NHS Business Services authority. Dental Services. Digest Search. NHSBSA. Online search available at: http://www.nhsbsa.nhs.uk/DentalServices/2876.aspx (accessed August 2012)

10. Statement of dental remuneration 2005. Online article available at http://www.nhsbsa.nhs.uk/ Documents/ArchivePDF/sdr93.pdf (accessed August 2012).

11. Sheldon T A, Cullum N, Dawson D et al. What's the evidence that NICE guidance has been implemented? Results from a national evaluation using time series analysis, audit of patients' notes, and interviews. BMJ 2004; 329: 999.

12. University of York. Effectiveness matters. Prophylactic removal of impacted third molars: is it justified? NHS Centre for reviews and dissemination, University of York 1998; 3.

13. Channel 4 Dispatches. The truth about your dentist UK [television], 23 May 2011.

14. McArdle L W. Oral surgery referral patterns 2004/2005 - 2009/2010. London: Internal Service Evaluation Report, Guy's \& St Thomas's NHS Foundation Trust, 2010.

15. Bruce R A, Frederickson G C, Small G S. Age of patients and morbidity associated with mandibular third molar surgery. J Am Dent Assoc 1980; 101: 240-245

16. McArdle $L W$, Renton T F. Distal cervical caries in the mandibular second molar: an indication for the 
prophylactic removal of the third molar? Br J Oral Maxillofac Surg 2006; 44: 42-45.

17. Chang S W, Shin SY, Kum KY, Hong J. Correlation study between distal caries in the mandibular second molar and the eruption status of the mandibular third molar in the Korean population. Oral Surg Oral Med

Oral Pathol Oral Radiol Endod 2009; 108: 838-843.

18. Chu F C, Li T K, Lui V K, Newsome P R, Chow R L, Cheung L K. Prevalence of impacted teeth and associated pathologies - a radiographic study of the Hong Kong Chinese population. Hong Kong Med J 2003; 9: 158-163.

19. Adeyemo $W$ L, James 0, Ogunlewe M O, Ladeinde A L, Taiwo 0 A, Olojede A C. Indications for extraction of third molars: a review of 1763 cases. Niger Postgrad Med J 2008; 15: 42-46.

20. van der Linden W, Cleaton-Jones P, Lownie M. Diseases and lesions associated with third molars. Review of 1001 cases. Oral Surg Oral Med Oral Pathol Oral Radiol Endod 1995; 79: 142-145.

21. Knutsson K, Brehmer B, Lysell L, Rohlin M. Pathoses associated with mandibular third molars subjected to removal. Oral Surg Oral Med Oral Pathol Oral Radiol Endod 1996; 82: 10-17.

22. Brickley M R, Shepherd J P. An investigation of the rationality of lower third molar removal, based on USA National Institutes of Health criteria. Br Dent $J$ 1996; 180: 249-254.
23. Nordenram $\AA$, Hultin M, Kjellman O, Ramström G Indications for surgical removal of the mandibular third molar. Study of 2,630 cases. Swed Dent J 1987; 11: 23-29.

24. Lysell L, Rohlin M. A study of indications used for removal of the mandibular third molar. Int J Oral Maxillofac Surg 1988; 17: 161-164.

25. Office for National Statistics. Adult Dental Health Survey, 1998. London: HMSO, 2000.

26. Office for National Statistics. Adult Dental Health Survey, 2009. London: HMSO, 2011.

27. Thomas D, Walker R, Smith A, Shepherd J. The provision of OS services in England and Wales 1984-1991. Br Dent J 1994; 176: 215-219. 